\title{
CONHECENDO A SAÚDE NUTRICIONAL DE IDOSOS ATENDIDOS EM UMA ORGANIZAÇÃO NÃO GOVERNAMENTAL, BENEVIDES / PA
}

\section{GETTING TO KNOW THE NUTRITIONAL HEALTH OF ELDERLY PEOPLE ASSISTED BY A NON-GOVERNMENTAL ORGANIZATION, BENEVIDES / PA}

Rozinéia de Nazaré Alberto Miranda * Elenilce Pereira Carvalho**

Yane Ramos Amorim**** Kelly Santos dos Santos***** Fernanda Oliveira Serrão*****

\section{RESUMO}

Este artigo teve como objetivo descrever o perfil socioeconômico, antropométrico, dietético e identificar a prevalência de doenças crônicas em idosos. Foi um estudo transversal, descritivo realizado com idosos atendidos pelo Projeto de Extensão "Nutrição e Saúde na 3a idade", Faculdade de Nutrição/UFPA em uma Organização Não Governamental. Foram coletadas através de um questionário semiestruturado variáveis socioeconômicas e de estilo de vida, presença ou não de doenças crônicas, varáveis antropométricas e consumo alimentar. 185 idosos foram avaliados, predomínio do sexo feminino na faixa etária de 60 a 69 anos. Detectou-se em sua maioria a presença acentuada de sedentarismo e de um estado nutricional de sobrepeso/obesidade, segundo o índice de massa corpórea; as outras medidas compartimentadas apresentaram eutrofia, prevalência de hipertensão arterial e diabetes mellitus e grande ingestão de carboidratos simples e baixo consumo de alimentos protetores. Assim, todos esses dados direcionam os idosos para um perfil indicativo de risco nutricional.

Palavras-chave: idosos; antropometria; dietética; doenças crônicas.

\footnotetext{
*Professora da Universidade Federal do Pará (UFPA), PA - Brasil. E-mail: rozi@ufpa.br

**Nutricionista do Hospital Universitário João de Barros Barreto - Universidade Federal do Pará (UFPA), PA - Brasil. E-mail: carvalhonut@hotmail.com

***Aluna de Graduação da Universidade Federal do Pará (UFPA), PA - Brasil. E-mail: yane_amorym@hotmail.com

****Aluna de Graduação da Universidade Federal do Pará (UFPA), PA - Brasil. E-mail: kellydejesus21@gmail.com 


\section{ABSTRACT}

This article aimed to describe the socioeconomic, anthropometric and dietary profile and identifying the prevalence of chronic diseases in elderly people. It was a cross - sectional and descriptive study carried out with elderly people assisted by the Extension Project "Nutrition and Health in $3^{\text {rd }}$ age", College of Nutrition/Federal University of Pará in a Non-Governmental Organization. A semistructured questionnaire was used to collect socioeconomic and lifestyle variables. The presence or not of chronic diseases was assessed through anthropometric measurements and food intake evaluation. 185 elderly were evaluated, with a predominance of women aged 60-69 years. High presence of sedentary lifestyle, nutritional status of overweight or obesity according to body mass index and other compartmental measures surveyed showed eutrophy, prevalence of arterial hypertension and diabetes mellitus along with a large intake of simple carbohydrates and low consumption of protective foods. Thus, all these data pointed out the elderly's indicative profile of nutritional risk.

Keywords: elderly; anthropometry; dietetics; chronic diseases.

\section{Introduçáo}

A queda da mortalidade e o aumento da natalidade nos países industrializados, iniciada no século passado, aconteceram juntamente com a ampliaçáo da cobertura de vacinas, do sistema de proteçáo social e das melhorias nas condiçóes habitacionais, de alimentação, de saneamento básico e de trabalho, fazendo com que a populaçáo idosa no mundo se tornasse numerosa (SOUZA, BRANCA, 2011).As taxas de fecundidade nos países em desenvolvimento caíram possibilitando que, ao longo de apenas uma geração, diversos países deixem de possuir uma população jovem para terem um maior número de idosos. Essas modificaçôes nas características da população vêm alterando o perfil epidemiológico no Brasil, que passará do estágio de jovem (até 7\% de idosos) para envelhecido (mais de 14\% de idosos) em apenas 25 anos - entre 2011 e 2036 (MORAES, 2012).

O processo de envelhecimento traz consigo diversas modificações, seja em níveis fisiológicos, bioquímicos, psicológicos e funcionais, além dosaspectos sociais, que tornam os idosos mais suscetíveis a doenças, principalmente as de caráter crônico-degenerativas, afetando diretamente o estado nutricional desses indivíduos (TIRAPEGUI; RIBEIRO, 2011; PAZ; FAZZIO, SANTOS, 2012; NASCIMENTO et al., 2011).

As doenças crônicas não transmissíveis (DCNT), de acordo com a OMS (2005), são principalmente as doenças cardiovasculares, em especial as cardíacas, os acidentes vasculares cerebrais, câncer, diabetes e doenças respiratórias crônicas, que apresentam um curso clínico que se modifica gradualmente no decorrer da vida; elas são de longa duração (gerando maior procura pelos serviços de saúde), o que fazcom que haja um aumento na mortalidade e nas incapacidades funcionaisdo indivíduo. Isto traz consigo alguns desafios, pois acarreta em novas demandas na área da saúde (CARNEIRO et al. 2013; MALTA; SILVA JR., 2013; BRASIL, 2014; FREIRE et al., 2015).

Devido à magnitude dessas enfermidades e ao fato de seus fatores de risco serem comuns a outras doenças crônicas, o Ministério da Saúde implementou políticas de combate, priorizando açóes de alimentação saudável, atividade física, prevenção ao uso de tabaco e álcool,a fim de combater o crescimento deste grupo de doenças (BRASIL, 2014).A avaliação nutricionalmente é uma ferramenta importante para diagnosticar precocemente os distúrbios nutricionais próprios de cada fase da vida, como a desnutriçāoo e a obesidade, que trazem vinculadas outras doenças crônicas e agudas, as quais podem acelerar o desenvolvimento de doenças degenerativas (PAZ; FAZZIO; SANTOS, 2012; RIBEIRO et al., 2011). 
De acordo com Paz, Fazzio e Santos (2012), a avaliaçáo nutricional da população idosa é complexa devido às modificações decorrentes do envelhecimento. $\mathrm{O}$ declínio de massa magra e líquidos corpóreos, aumento da quantidade de tecido adiposo e diminuiçáo de alguns órgáos são alguns dos aspectos observados no idoso, o que faz com que se necessitem de profissionais experientes para realizar o diagnóstico nutricional e traçar condutas dietéticas que visem à melhora da qualidade de vida deste grupo (SILVA et al., 2010).

Este trabalho foi desenvolvido com o objetivo de conhecer o perfil antropométrico e alimentar de idosos, além de identificar a prevalência de doenças crônicas, atendidos em uma Organizaçáo Não Governamental, localizada no município de Benevides- PA, a qual é atendida pelo Projeto de Extensão "Nutriçáo e Saúde na 3a idade", da Faculdade de Nutrição da Universidade Federal do Pará.

\section{Métodos}

\section{Caracterização do estudo}

Esta pesquisa foi feita a partir do modelo transversal, aleatório, de características descritivas e analíticas, realizada no período de junho de 2015 a setembro de 2016. Foi aplicado um questionário semiestruturado para obtençáo das seguintes variáveis:identificaçáo (sexo, data do nascimento, etc.), estilo de vida(ingestáo de bebida alcoólica, hábito de fumar, prática de atividade física) e a presença ou não de doenças crônicas náo degenerativas, dados que costumam influenciar questóes relacionadas à saúde.

$\mathrm{Na}$ antropometria, foi mensurado peso $(\mathrm{Kg})$ e altura $(\mathrm{m})$ para obtençáo do índice de massa corporal (IMC), dobra cutânea tricipital (PCT), circunferência do braço (CB), circunferência muscular do braço $(\mathrm{CMB})$ e circunferência da panturrilha $(\mathrm{CP})$. As técnicas de aferiçấo das medidas antropométricas seguiram os preceitos estabelecidos por Lohman (1988).

As medidas de peso e altura foram realizadas em balança e analisador corporal digital científico Wiso Care W721, com medidor de altura por ultrassom, capacidade para $180 \mathrm{~kg}$ e precisão de $100 \mathrm{~g}$. Os pacientes foram pesados sem sapatos e com roupas leves. A altura foi medida com o paciente em posição ereta, com os pés juntos, olhar direcionado para um ponto fixo, sem calçados ou adornos na cabeça (SISVAN, 2004).

A PCT foi mensurada utilizando adipômetro científico digital Prime Vision Med com capacidade de 0 a $60(\mathrm{~mm})$ e precisão de $0,1(\mathrm{~mm})$, mensurando-se três medidas e adotando a média como valor final. Para aferir essamedida foi necessário marcar o ponto médio do braço, entre o acrômio e o olécrano e pinçar o tecido subcutâneo na parte posterior do braço paralelamente ao eixo longitudinal. A PCT foi adequada segundo tabela de percentis da NHANES III, a partir da fórmula PCT (\%) = PCT obtida $(\mathrm{mm}) \times 100 /$ PCT percentil 50 e classificação de acordo com Blackburn e Thornton (BUSNELLO, 2007).

A CB foi verificada utilizando-se fita métrica inelástica com precisão de 1 milímetro: o avaliado permaneceu de pé e o braço dominante foi flexionado, formando ângulo de 90 graus. Em seguida, foi marcado o ponto médio entre o acrômio e o olecrano,solicitando após issoque o braço ficasse relaxado e a palma da mão voltada para a parte interna do 
corpo; a fita métrica contornou o braço no ponto demarcado, e a medida realizada sem exercer pressão sobre o braço. Através da $\mathrm{CB}$ classificou-se o estado nutricional, segundo a tabela de percentis da NHANES III adequada pela equaçáoCB $(\%)=\mathrm{CB}$ obtida $(\mathrm{mm})$ x 100 / CB percentil 50 e classificada segundo Blackburn e Thornton (TIRAPEGUI; RIBEIRO, 2011).

A estimativa da circunferência muscular do braço $(\mathrm{CMB})$ foi realizada a partir da equação $\mathrm{CMB}(\mathrm{cm})=\mathrm{CB}(\mathrm{cm})-\pi \mathrm{x}[\mathrm{PCT}(\mathrm{mm}) \div 10]$, obtida a partir dos valores da $\mathrm{CB}$ e PCT. A CMB avalia a reserva de tecido muscular sem correção da área óssea. A medida foi adequada pela equação $\mathrm{CMB}(\%)=\mathrm{CMB}$ obtida $(\mathrm{mm}) \times 100 / \mathrm{CB}$ percentil 50 , utilizando percentis segundo faixas etárias proposto por NHANESIII (BUSNELLO, 2007).

A circunferência da panturrilha foi mensurada na perna esquerda, com uma fita métrica inelástica, na sua parte mais protuberante. Valores inferiores a 31 centímetros indicam perda de massa muscular (WHO, 1995).

$\mathrm{Na}$ dietética, a identificação dos hábitos alimentares foi conhecida a partir da aplicação do Questionário de Frequência Alimentar (QFA), segundo Fisberg et al. (2008), por grupos de alimentos, adaptado de acordo com a regionalidade e agrupados em três grandes grupos de alimentos: energéticos, proteicos e reguladores. Sua análise foi feita de acordo com as recomendaçóes do Guia Alimentar da População Brasileira (2014).

A seguinte pesquisa seguiu os princípios éticos que dizem respeito à autonomia das pessoas envolvidas, segundo Resolução 466 do Conselho Nacional de Saúde, 2012. O projeto foi aprovado pelo Comitê de Ética em Pesquisa do Instituto de Ciências da Saúde da Universidade Federal do Pará - ICS/UFPA (parecer no 1.179.249). Todos os participantes leram e assinaram o Termo de Consentimento Livre e Esclarecido.

\section{Análise estatística}

As análises estatísticas dos dados foram realizadas com o auxílio dos softwares Microsoft Office Excel e o programaBioEstat 5.0 (AYRES et al., 2007).As estimativas das prevalências foram apresentadas em proporçóes (\%), com seus respectivos intervalos de confiança de $95 \%$ (IC95\%).

A análise de variância (ANOVA) e a comparação de Tukey foram utilizadas para verificar o efeito do grupo etário nas variáveis antropométricas, quando a amostra apresentava normalidade, e o teste de Kruskal-Wallys para dados com distribuição não normal. O nível de significância foi fixado em $\mathrm{p}<0,05$.

\section{Caracterizaçáo do projeto de extensáo}

O projeto de extensão "Nutriçáo e Saúde na 3a idade", da Faculdade de Nutrição da Universidade Feral do Pará (UFPA), está vinculado ao edital no 11/2015, Eixo Transversal/2015 - Recursos naturais: Ciência, Direito e Realidade, e tem como objetivo principal o conhecimento do perfil epidemiológico, nutricional e alimentar de idosos, frequentadores de uma Organização Não Governamental (ONG) do município de Benevides-PA. O trabalho foi contemplado com uma bolsa de extensão e também possui uma estagiária voluntária, levando o discente a colocar em prática os conhecimentos 
teóricos adquiridos na faculdade. $\mathrm{O}$ atendimento é realizado todas as quartas-feiras, das $8 \mathrm{~h}$ às $12 \mathrm{~h}$.

Além da obtenção dos princípios básicos, o projeto realiza atividades paralelas: educaçáo nutricional, atendimento individualizado aos portadores de doenças crônicas preexistentes, palestras educativas, atividades lúdicas acerca da saúde e nutrição dos idosos, visando sempre diminuir o abismo de conhecimento na comunidade.

\section{Resultados}

A população em estudo foi composta por 185 idosos com idade igual ou acima de 60 anos, frequentadores do "Projeto Nutrição e Saúde na 3a Idade", em Benevides - Pa. Para os fins deste estudo, os idosos foram agrupados em três faixas etárias: idosos jovens (60 a 69 anos), idosos medianos (70 a 79 anos) e idosos longevos (com 80 ou mais anos).

$\mathrm{Na}$ Tabela 1 são apresentadas as variáveis de identificação dos idosos, bem como o estilo de vida e presença de doenças crônicas não transmissíveis. Observa-se prevalência do sexo feminino $(78,92 \%)$, com predomínio na faixa etária dos 70 a 79 anos $(80,7 \%$; $\left.\mathrm{IC}_{95 \%} 70,2-87,7\right)$. Os idosos em sua maioria encontraram-se na faixa etária de 60 a 69 anos $(71,89 \%)$.

Em relação ao estado civil, 40,70\% dos idosos jovens são solteiros $\left(\mathrm{IC}_{95 \%}\right.$ 31,9-46,9). $\mathrm{O}$ estado de viuvez acometeu as demais faixas etárias, sendo predominante no grupo dos com mais de 80 anos $\left(66,66 \%\right.$ IC $\left._{95 \%} 40,0-86,7\right)$. $\mathrm{Na}$ situação profissional,em todas as faixas etárias predominaram os idosos aposentados, com prevalência entre os longevos $\left(86,66 \% ; \mathrm{IC}_{95 \%} 66,7-92,9\right)$.

A situaçẫo econômica, em todos os grupos etários, foi de predomínio de renda mensal de 1-3 salários mínimos, sendo que na faixa etária dos idosos medianos a prevalência foi maior $\left(85,96 \% \mathrm{IC}_{95 \%} 77,2-91,2\right)$.

Quanto ao uso de tabaco, 7,56\% (n= 14) são fumantes ativos e 33,51\% ( $\mathrm{n}=62)$ extabagistas, a ingestấo de bebida alcoólica se fez presente ocasionalmente em 21,08\% ( $\mathrm{n}=$ 39). Em relação à prática de atividade física, $44,86 \%(\mathrm{n}=83)$ não realiza nenhum tipo de exercício físico, sendo o grupo de idosos longevos os mais fisicamente inativos (66,66\%; $\left.\mathrm{IC}_{95 \%} 40,0-86,7\right)$.

Dentre as comorbidades, a mais recorrente foi a hipertensão arterial sistêmica (41,62\%), seguida da diabetes (23,24\%), osteoporose (18,3\%) e acidente vascular cerebral - AVC (5,95\%). A diabetes se manifestou no grupo de idosos medianos (31,57\%, $\left.\mathrm{IC}_{95 \%}: 19,3-40,4\right)$, enquanto a hipertensão predominou no grupo dos com mais de 80 anos $\left(60 \%\right.$; $\left.\mathrm{IC}_{95 \%} 33,3-80,0\right)$. Na faixa etária de 60 a 69 anos, $11,50 \%\left(\mathrm{IC}_{95 \%} 5,3-16,8\right)$ já apresentaram AVC e, entre os de 70 a 79 anos, 29,82\% ( $\left.\mathrm{IC}_{95 \%} 17,6-40,4\right)$ já tiveram osteoporose. 
Tabela 1: Variáveis socioeconômicas, estilo de vida e presença de doenças crônicas náo transmissíveis do "Projeto Nutrição e Saúde na 3a Idade”, Benevides - Pa

\begin{tabular}{|c|c|c|c|c|c|c|}
\hline \multirow[t]{2}{*}{ VARIÁVEIS } & \multicolumn{2}{|c|}{60 a 69 anos } & \multicolumn{2}{|c|}{70 a 79 anos } & \multicolumn{2}{|c|}{$\geq 80$ anos } \\
\hline & $\%$ & IC95\% & $\%$ & IC95\% & $\%$ & IC95\% \\
\hline \multicolumn{7}{|l|}{ Sexo } \\
\hline Feminino & 79,6 & $71,7-85,9$ & 80,7 & $70,2-87,7$ & 66,7 & $40,0-86,7$ \\
\hline Masculino & 20,4 & $13,3-26,6$ & 19,3 & $0,00-36,4$ & 33,3 & $13,3-46,7$ \\
\hline \multicolumn{7}{|l|}{ Estado Civil } \\
\hline Solteiro (a) & 40,70 & $31,9-46,9$ & 22,80 & $12,3-31,6$ & 6,66 & $40,0-86,7$ \\
\hline Casado (a) & 26,54 & $18,6-33,6$ & 33,33 & $21,1-42,1$ & 26,66 & $6,7-40,0$ \\
\hline Viúvo (a) & 32,74 & $24,8-39,8$ & 43,85 & $29,8-54,4$ & 66,66 & $40,0-86,7$ \\
\hline \multicolumn{7}{|l|}{ Situação Profissional } \\
\hline Aposentado & 54,86 & $45,1-62,8$ & 78,94 & $68,4-87,7$ & 86,66 & $66,7-92,9$ \\
\hline Não aposentado & 30,0 & $22,1-36,3$ & 3,50 & $22,8-43,9$ & - & - \\
\hline Pensionista & 15,0 & $8,9-20,4$ & 17,54 & $8,8-24,6$ & 13,33 & $0,00-20,0$ \\
\hline \multicolumn{7}{|l|}{ Renda } \\
\hline$<1 \mathrm{SM}$ & 21,23 & $13,3-27,4$ & 10,52 & $3,5-15,8$ & 13,33 & $0,00-20$ \\
\hline $1-3 \mathrm{SM}$ & 78,76 & $71,7-84,1$ & 85,96 & $77,2-91,2$ & 73,33 & $46,7-93,3$ \\
\hline$>3 \mathrm{SM}$ & - & - & 3,50 & $22,8-45,6$ & 13,34 & $0,00-20,0$ \\
\hline \multicolumn{7}{|l|}{ Tabagismo } \\
\hline Fumante & 8,86 & $0,44-12,4$ & 7,03 & $1,8-10,5$ & - & - \\
\hline Ex-fumante & 31,85 & $23,0-38,9$ & 35,08 & $22,8-43,9$ & 40,0 & $13,3-60,0$ \\
\hline Nunca fumou & 59,29 & $50,5-66,6$ & 57,89 & $45,6-68,4$ & 60,0 & $33,3-80,0$ \\
\hline \multicolumn{7}{|l|}{ Etilismo } \\
\hline $\begin{array}{l}\text { Não ingere bebidas } \\
\text { alcoólicas }\end{array}$ & 81,41 & $74,3-87,6$ & 73,68 & $59,7-84,2$ & 80,0 & $60,0-93,3$ \\
\hline Ocasionalmente & 18,58 & $11,5-24,8$ & 26,31 & $15,8-35,1$ & 20,0 & $0,00-40,0$ \\
\hline $\begin{array}{l}\text { Mais de três vezes por } \\
\text { semana }\end{array}$ & - & - & - & - & - & - \\
\hline \multicolumn{7}{|l|}{ Atividade Física } \\
\hline Pratica & 27,43 & $19,5-33,6$ & 17,54 & $8,8-24,6$ & 6,66 & $0,00-6,7$ \\
\hline Não pratica & 41,59 & $32,8-48,7$ & 45,61 & $31,6-56,2$ & 66,66 & $40,0-86,7$ \\
\hline Ocasionalmente & 30,97 & $23,0-38,1$ & 36,84 & $26,3-45,6$ & 26,66 & $6,7-40,0$ \\
\hline \multicolumn{7}{|l|}{ Doenças Crônicas } \\
\hline Diabetes & 23,89 & $15,9-31,0$ & 31,57 & $19,3-40,4$ & 26,66 & $6,7-46,7$ \\
\hline Hipertensão & 42,47 & $32,8-50,5$ & 42,10 & $29,8-50,9$ & 60,0 & $33,3-80,0$ \\
\hline AVC & 11,50 & $5,3-16,8$ & 3,50 & $22,8-45,6$ & - & - \\
\hline Osteoporose & 22,14 & $15,1-28,3$ & 29,82 & $17,6-40,4$ & 13,33 & $0,00-0,20$ \\
\hline
\end{tabular}

IC: Intervalo de Confiança; SM: Salário Mínimo; AVC: Acidente Vascular Cerebral.

A mulher brasileira possui uma expectativa de vida de aproximadamente oito anos a mais que o homem (IBGE, 2012), podendo-se inferir que essa longevidade está ligada a uma questáo cultural, uma vez que as mulheres tendem a procurar mais os serviços oferecidos para melhorar sua qualidade de vida, enquantoos homens, em geral, cuidam-se menos, principalmente se tratando da saúde pessoal (FREIRE et al., 2015).

A pesquisa em questáo obteve resultados semelhantes ao encontrado nos estudos de Sobreira et al. (2011), uma vez que o grupo de convivência foi composto principalmente por mulheres (87\%), o que reporta a preocupação maior que as mulheres possuem em preencher seu cotidiano com atividades que trazem benefícios à sua saúde de um modo geral (física, psicológica e social). 
A prevalência de idosos na faixa etária de 60 a 69 anos neste estudo (71, 89\% $\mathrm{n}=113$ ) remete à maior capacidade física que eles apresentam para realização de suas atividades de vida diária, ainda que seja observada a presença de alguma doença crônica. Segundo Borges et al. (2010), a menor participação de idosos acima de 80 anos em programas sociais decorre em funçáo dadependência familiar para a execuçáo de suas atividades de vida diária.

Foi constatado na pesquisa um reduzido número de idosos ativos que ainda utilizam o fumo frequentemente, mesmo assim foi enfatizado,por meio do processo de educação em saúde realizado no projeto, que o tabagismo é um fator agravante para as doenças do idoso, sendo o mesmo responsável por várias comorbidades. Os benefícios para a saúde,que a suspensáo do hábito de fumar acarreta, sáo evidentes em todas as faixas etárias, sendo imprescindível que ocorra essa mudança no estilo de vida(GOULART et al., 2010).

O consumo de álcool encontrado entre os idosos corroborou os resultados do estudo de Sobreira et al., (2011), já que a maior parte dos idosos pesquisados respondeu náo fazer mais uso de bebida alcoólica. $\mathrm{O}$ estilo de vida pode ser modificado para ser um fator protetor da saúde, pois foi reportado pela maioria dos pesquisados que em sua juventude fizeram uso de bebidas alcóolicas.

A maioria dos idosos não pratica atividade física regular, ainda que a $\mathrm{ONG}$, onde o Projeto de Extensão está inserido, ofereçaa oportunidade. Esse índice insatisfatório é preocupante, considerando que a inatividade física pode potencializar e indicarriscocardiovascular, quando comparados com indivíduos ativos (RIBAS, 2013). Dessa forma, o exercício físico pode e deveser encarado como uma das principaisferramentas do processo saúde/doença, pois traz contribuiçóes essenciais durante a terceira idade; necessita-se de um maior engajamento dos órgáos governamentais para que atuem como incentivadores na adoção de práticas saudáveis (ZAGO, 2010).

A patologia mais citada entre os idosos foi a hipertensão arterial sistêmica (41,62\%), resultado muito próximo (47\%) do encontrado nos estudos de Hott e Pires (2011). Essa doença, segundo o volume VI das Diretrizes Brasileiras de Hipertensão Arterial Sistêmica (HAS), aponta o sedentarismo como responsável pelo aumento da sua incidência, levando a um importante aumento nos eventos cardiovasculares com consequente diminuição da sobrevida e piora na qualidade de vida dos sujeitos. Assim, as principais recomendaçóes não medicamentosas para prevenção primária da HAS devem ser adotadas através dos hábitos saudáveis de vida: alimentação equilibrada, consumo controlado de sódio, álcool, combate ao sedentarismo e ao tabagismo (SBH, 2010).

Diabetes mellitus $(\mathrm{DM})$ não é uma única doença, mas um grupo heterogêneo de distúrbios metabólicos. Sua prevalência é maior nos idosos quando comparado com a população geral, podendo atingir cerca de $20 \%$ das pessoas com 75 a 80 anos. Esse fato pode ser entendido por várias razóes, a exemplo do aumento da expectativa de vida com concomitante redução de algumas funçôes orgânicas, que auxiliam no controle das taxas de glicose - secreção de insulina e sensibilidade à ação da insulina, funçôes responsáveis pela utilizaçáo da glicose pelas células(SBD, 2016).

No estudo de Francisco et al. (2010), a diabetes mellitus foi citadapor 15,4\% dos idosos, resultado maior foi encontrado nesta pesquisa (23,24\%). Dentre os fatores de risco para o aparecimento dessas doenças,podemos citar hipertensão arterial, excesso de peso e o sedentarismo, variáveis observadas e referenciadas nesta pesquisa. 
Pode-se afirmar, portanto, que a modificação no estilo de vida é um ponto importante a ser trabalhado no manejo do diabético idoso (MENDES et al., 2011). Em um estudo realizado em 2015, que utilizou dados de 2013 do Sistema Único de Saúde, Bielemann (2015) revelou que a taxa de internaçóes hospitalares para diabetes e hipertensão predominaram na regiáo Norte (257,5 e 221,3 internaçóes/100.000 habitantes, respectivamente), sendo que $37 \%$ das internaçôes por diabetes foram relacionadas à inatividade física.

A osteoporose é uma doença sistêmica e progressiva caracterizada por uma redução na massa óssea e desarranjo de sua microarquitetura. A prática de atividade física regular, aliada a uma alimentaçáo saudável e equilibrada, influencia diretamente no retardo do aparecimento dessa doença. Silveira et al. (2011) em seus estudos encontraram a osteoporose em $25 \%$ dos idosos pesquisados, resultado inferior, mas ainda preocupante, desteestudo (18,38\%), considerando queas sequelas clínicas, como a debilidade dos doentes, perda de sua dependência e o aumento da mortalidade são grandes no grupo populacional da terceira idade.

Segundo o Ministério da Saúde (BRASIL, 2013), o AVC decorre da insuficiência no fluxo sanguíneo em uma determinada área do cérebro e tem diferentes causas.O tratamento engloba a adoção de medidas preventivas, controlandovários fatores de risco que são comumente encontrados na população geriátrica, como a hipertensão arterial, diabetes e o tabagismo (CARVALHO et al., 2015).

$\mathrm{Na}$ geriatria, a avaliação do estado nutricional é complexa devido às alteraçóes que ocorrem no envelhecimento, devendo-se levar em consideraçáo, assim, a particularidade de cada idoso. O estado nutricional possui implicaçóes importantes no contexto do envelhecimento, devido ao fato de que o controle de grande parte das doenças crônicas e/ou infecciosas e a prevençáo de suas complicaçóes dependem do estado nutricional do indivíduo (NASCIMENTO et al., 2011; PAZ; FAZZIO; SANTOS, 2012).

Dentre as várias formas de avaliar o estado nutricional de idosos, as medidas antropométricas apresentam-se como as de maior utilizaçáo, sendo um método não invasivo, de baixo custo, fácil e de rápida execução, permitindo boa correlação com indicadores de morbimortalidade (CORTEZ; MARTINS, 2012; PEREIRA; SPYRIDES; ANDRADE, 2016).A classificação das variáveis antropométricas, segundo a faixa etária dos idosos, pode ser encontrada na Tabela 2. A população estudada caracteriza-se por apresentar predominância de sobrepeso, de acordo com o IMC, desnutrição, segundo a PCT, e eutrofia por meio da análise das medidas da CB, CMB e CP. Observou-se que as médias dos valores de IMC sofreram declínio com o avançar da idade, masessa redução não foi estatisticamente significativa ( $p>0,05)$. 


\section{Tabela 2: Classificaçáo das variáveis antropométricas segundo faixa etária em idosos do "Projeto Nutriçáo e Saúde na $3^{a}$ Idade", Benevides - Pa}

\begin{tabular}{|c|c|c|c|c|c|c|c|}
\hline Variáveis & & Faixas & & Etárias & & & \\
\hline & $\mathbf{N}$ & $\begin{array}{l}60-69 \\
M \pm D P\end{array}$ & $\mathbf{N}$ & $\begin{array}{l}70-79 \\
M \pm D P\end{array}$ & $\mathbf{N}$ & $\begin{array}{c}\geq 80 \text { anos } \\
M \pm D P\end{array}$ & $p$ valor \\
\hline \multicolumn{8}{|l|}{ IMC } \\
\hline Desnutrição & 13 & $20,83 \pm 1,31$ & 7 & $20,22 \pm 1,47$ & 1 & $18 \#$ & \\
\hline Eutrofia & 38 & $25,65 \pm 1,86$ & 22 & $25,18 \pm 1,19$ & 6 & $24,40 \pm 1,33$ & 0,32 \\
\hline Sobrepeso & 62 & $31,49 \pm 3,04$ & 28 & $31,29 \pm 2,17$ & 8 & $30,86 \pm 1,62$ & \\
\hline Total & 113 & $28,50 \pm 3,78$ & 57 & $27,58 \pm 3,65$ & 15 & $27,42 \pm 3,68$ & \\
\hline \multicolumn{8}{|l|}{ PCT } \\
\hline Desnutrição & 75 & $14,71 \pm 4,17$ & 39 & $13,25 \pm 3,91$ & 8 & $11,60 \pm 2,93$ & $0,015^{*}$ \\
\hline Eutrofia & 22 & $19,35 \pm 4,93$ & 11 & $18,86 \pm 4,29$ & 3 & $16,00 \pm 2,66$ & \\
\hline Sobrepeso & 16 & $23,08 \pm 5,18$ & 7 & $28,64 \pm 3,13$ & 4 & $22,19 \pm 4,59$ & \\
\hline Total & 113 & $17,01 \pm 5,12^{a}$ & 57 & $16,56 \pm 5,3$ & 15 & $15,31 \pm 4,7^{\mathrm{a}}$ & \\
\hline \multicolumn{8}{|l|}{ CB } \\
\hline Desnutrição & 36 & $26,29 \pm 1,61$ & 14 & $25,92 \pm 2,33$ & 4 & $22,50 \pm 3$ & $>0,05$ \\
\hline Eutrofia & 59 & $30,83 \pm 1,41$ & 33 & $29,88 \pm 0,97$ & 9 & $30,21 \pm 1,34$ & \\
\hline Sobrepeso & 18 & $37,32 \pm 2,89$ & 10 & $35,41 \pm 2,18$ & 2 & $31,6 \pm 0$ & \\
\hline Total & 113 & $30,42 \pm 3,19$ & 57 & $29,88 \pm 2,7$ & 15 & $28,34 \pm 3,21$ & \\
\hline \multicolumn{8}{|l|}{ CMB } \\
\hline Desnutrição & 23 & $22,16 \pm 2,16$ & 9 & $20,53 \pm 1,91$ & 3 & $18,63 \pm 2,69$ & $>0,05$ \\
\hline Eutrofia & 90 & $25,78 \pm 2,35$ & 48 & $25,3 \pm 2,04$ & 12 & $25,15 \pm 1,88$ & \\
\hline Total & 113 & $25,05 \pm 2,46$ & 57 & $24,55 \pm 2,33$ & 15 & $23,85 \pm 2,47$ & \\
\hline \multicolumn{8}{|l|}{ CP } \\
\hline Desnutrição & 13 & $26,98 \pm 0,7$ & 13 & $28,93 \pm 1,56$ & 5 & $29,14 \pm 0,91$ & $0,01^{*}$ \\
\hline Eutrofia & 100 & $35,41 \pm 2,25$ & 44 & $35,13 \pm 1,57$ & 10 & $34,23 \pm 1,45$ & \\
\hline Total & 113 & $34,88 \pm 2,51^{a}$ & 57 & $33,72 \pm 2,58$ & 15 & $32,53 \pm 2,42^{\mathrm{a}}$ & \\
\hline
\end{tabular}

IMC: índice de massa corporal; PCT: prega cutânea triciptal; CB: circunferência braquial; CMB: circunferência muscular do braço; CP: circunferência da panturrilha: \# não há desvio padrão, apresenta somente um indivíduo; * diferença estatisticamente significativa dos valores médios entre os grupos etários (ANOVA/Kruskal-Wallys); ${ }^{a}$ diferenças significativas entre as faixas etárias (Teste de Tukey/Dunn).

A prevalência de sobrepeso observada em todas as faixas etárias na pesquisa difere do resultado encontrado por Paz, Fazzio e Santos (2012) em seu estudo com idosos institucionalizados, em que $37,5 \%$ foram diagnosticados com desnutrição;. Todavia, os dados se assemelham ao estudo de Scherer (2013), que avaliou idosos de um grupo de convivência, sendo observada aprevalência de sobrepeso nos sujeitos analisados.

Sobrepeso e obesidade são sérios fatores de risco para o desenvolvimento de doenças crônicas, como as cardiovasculares e as diabetes. $\mathrm{O}$ acúmulo de gordura corporal pode acometer diferentes sistemas, associando-se a problemas metabólicos, sanguíneos, urinários, respiratórios e ósseos, além de tornar o idoso mais suscetivel a limitaçóes de mobilidade (SANTOS et al., 2013; SCHERER, 2013).

Nascimento et al. (2011) descreveram que há uma prevalência de baixo peso em idosos até os 79,9 anos de idade, reduzindo-se a partir dos 80 anos; essa perda de peso está relacionada a reduçáo do conteúdo de água corpórea, além de massa magra, o que pode explicar os valores observados na avaliação da PCT, principalmente na faixa etária de 70-79 anos (79,95\%), que detectaram diferenças estatisticamente significativas entre os grupos analisados $(\mathrm{p}=0,015)$. 
Os valores da $\mathrm{CB}$ mensurados mostraram que a maior parte dos idosos se encontravam eutróficos em todas as faixas etária e, quando combinados à PCT para o cálculo da circunferência muscular do braço (CMB), mostraram também o mesmo estado nutricional, sendo esta última a medida utilizada para diagnosticar alteraçóes da massa magra corporal (SANTOS et al., 2013). Os valores médios de ambas as variáveis declinaram àmedida que a idade aumentou, apesar de náo ter sido observado diferença estatística significante $(\mathrm{p}>0,05)$.

O envelhecimento humano é um processo biológico natural associado a diversas alteraçóes nos diferentes sistemas corporais existentes - cardíaco, respiratório, hormonal e musculoesquelético (FECHINE, TROMPIERI, 2012). Em relação a este último, no organismo humano há uma reduçáo de 10 a 16\% de massa muscular entre os 25 e 65 anos de idade, podendo chegar entre 40 e $50 \%$ em idades superiores há 60 anos, sendo essa reduçáo lenta e gradual. $\mathrm{O}$ resultado do estudo mostra a diminuiçáa gradativa de massa magra com o passar da idade, confirmando os estudos citados, podendo levar a uma série de alteraçóes e déficits funcionais e orgânicos, com perda progressiva de força e resistência muscular (ARAÚJO; BERTOLINI; JUNIOR, 2014).

A CP é uma medida sensível para avaliar a massa muscular e identificar a sarcopenia(SANTOS et al., 2013). Observou-se que a maioria dos idosos apresentou sua massa muscular preservada, sendo ainda reforçado pelos valores de CMB, em que os idosos apresentaram prevalência de eutrofia. Quando analisada entre as faixas etárias, observa-se que houve diferença significativa dos valores médios de CP $(\mathrm{p}=0,01)$, com predominância nas faixas etárias dos idosos jovens e dos longevos $(\mathrm{p}<0,05)$.

A diminuição das funçôes cognitivas e fisiológicas natural do envelhecimento é associada amodificaçóes no consumo alimentar, com alta ingestáo de calorias provenientes de gorduras, principalmente de origem animal, açúcar e alimentos que passam por processo de refinamento; esses são fatoresque podem contribuir para o excesso de peso corporal nos idosos, além dedesencadear ou colaborar para o agravamento de doenças já instaladas (ORLANDO et al., 2011; DOBNER; BLASI; KIRSTEN, 2012; MALTA, 2013).

Nos idosos avaliados, a prevalência de sobrepeso pode ser explicada, além dos fatores já citados acima, pelo consumo diário de alimentos energéticos, como o pão branco, a farinha de mandioca e o arroz branco, sendo este último consumido por $80 \%$ dos indivíduos (Gráfico 1), semelhante ao que se observa na população brasileira, em que o consumo de arroz foi de $84 \%$, computado através da Pesquisa de Orçamento Familiar (POF) 2008-2009 (IBGE, 2011). 


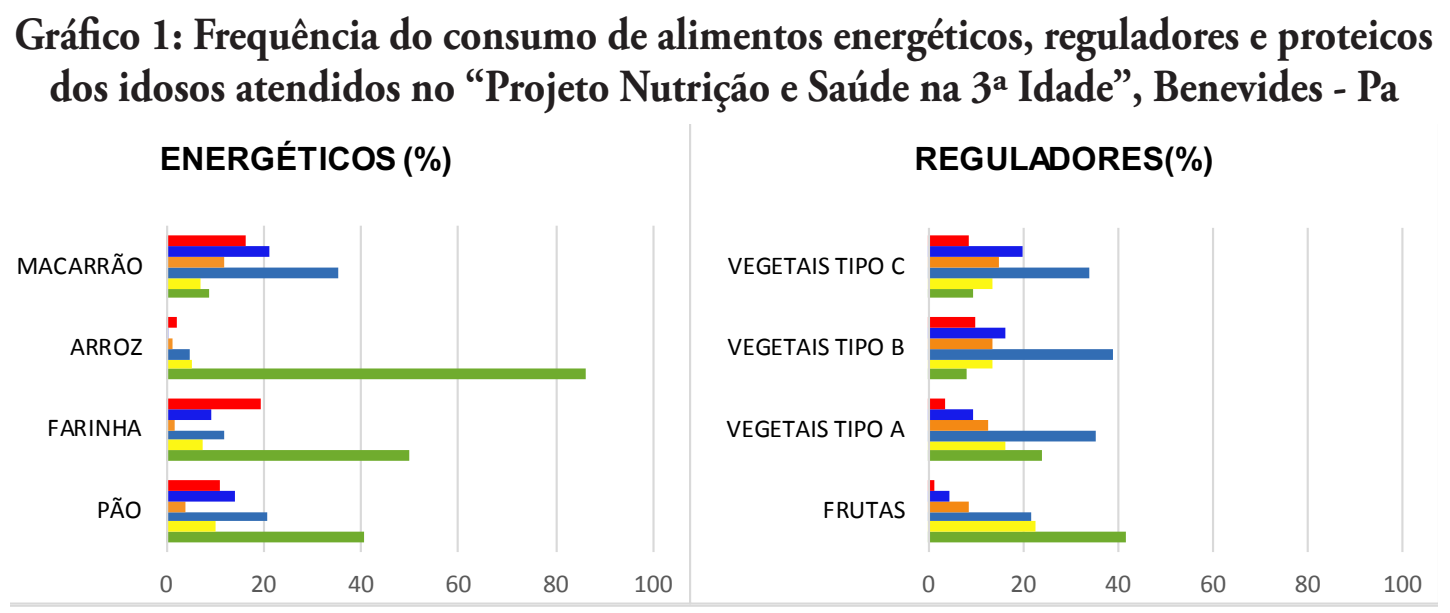

PROTEICOS (\%)

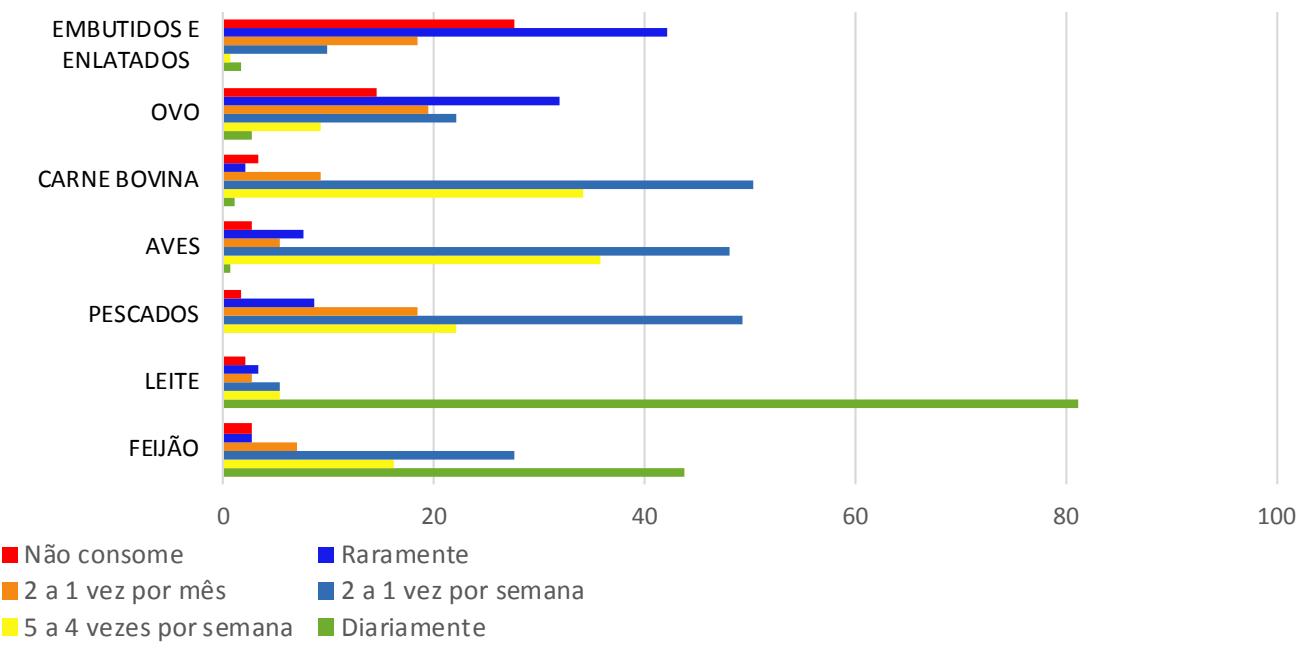

Os carboidratos são as principais fontes de energia para o organismo e esse macronutriente tende a ser mais consumido com o avançar da idade, por serde fácil aquisição, preparação e menor valor econômico, levando os idosos a optar pelo consumo na forma de caldos, sopas e mingaus, devido a problemas de dentiçãao e mastigação; isso também podelevá-los a uma baixa ingestáo de ferro (ORLANDO et al., 2011; FISBERG et al., 2013; VENTURINI et al., 2015).

Em relação aos alimentos reguladores, observou-se um baixo consumo, em consonância com o padrão observado pela POF (IBGE, 2011) na população brasileira. Apesardas frutas terem sido descritas como sendo consumidas diariamente, esses alimentos foram citados por menos de $50 \%$ dos idosos, com a referência a apenas um tipo de fruta.

A baixa ingestão de vegetais e frutas representa um fator de risco em relação ao desenvolvimento de doenças; o efeito positivo da fibra alimentar, encontrada nos alimentos reguladores, produz impacto sobre a velocidade do trato intestinal, reduçáo para o desenvolvimento de doença coronariana, hipertensáo, obesidade, diabetes e câncer de colón. Seria importante um consumo diário maior para obtençáo de todos esses benefícios, especialmente aquelas verduras e frutas fortemente coloridas, frescas, sendo ricas em vitaminas, minerais e fibras (TAVARES et al., 2015; MALTA; PAPINI; CORRENTE, 2013). 
Sobre as proteínas, o consumo diário de leite foi relatado por $81 \%$ dos idosos, seguido do feijão por 42\%. Segundo a POF (IBGE, 2011), na região Norte,esses alimentos são consumidos por apenas $6,9 \%$ e $57,7 \%$ da população, respectivamente, diferente do que foi encontrado na pesquisa. O leite é uma das principais fontes de cálcio, mineral que possui grande importância para os idosos, pois auxilia prevenindo o acometimento de doenças ósseas e suas consequências. Esse alimento é muito utilizado pelos idosos na composiçáo de mingaus, que muitas vezes substituem suas refeiçóes. Por sua vez, o feijáo é descrito como um dos alimentos que mais contribuem para o valor energético da dieta de idosos brasileiros, o que pode explicar o seu consumo entre $42 \%$ dos idosos avaliados (IBGE, 2011; DOBNER; BLASI; KIRSTEN, 2012; FISBERG et al., 2013).

Constatou-se que os idosos, em sua maioria, consomemcarnes (bovina, aves e peixes) duas a uma vez por semana, intercalando os tipos durante os dias - dados semelhantes aos encontrados por Panziera e colaboradores (2011) e também na POF (IBGE, 2011). Alimentos de origem animal são nutritivos, desde que sejam consumidos com moderaçáo, principalmente devido ao alto teor de gorduras saturadas, que podem aumentar o risco de desenvolvimento de obesidade, doenças cardíacas e outras patologias (ORLANDO et al., 2011).

Destaca-se a importância do baixo consumo (raro ou inexistente) de alimentos enlatados e embutidos entre os indivíduos desta análise (41\%), já que suaingestão excessiva contribui para o aumento da incidência de doenças crônicas não transmissíveis, como as cardiovasculares, síndrome metabólica e diversos tipos de câncer, como o gástrico e o colorretal (FREITAS et al., 2015).

A orientação nutricional deve ser um dos componentes da atenção à saúde da pessoa idosa, já que a alimentação saudável pode contribuir para a promoção de saúde e prevenção de doenças. Dessa forma, a populaçáo da terceira idade deve se motivada a buscar hábitos alimentares adequados, consumindo alimentos ricos em macro e micronutrientes, como os cereais integrais, frutas e hortaliças, que previnem tanto o baixo peso e a desnutrição quanto o sobrepeso e a obesidade (BRASIL, 2009; CAMARGOS et al. 2015; FISBERG et al., 2013).

\section{Conclusóes}

A presença acentuada de sedentarismo, estado nutricional de sobrepeso/obesidade em sua maioria, prevalência de hipertensão arterial e diabetes mellitus, grandes ingestôes de carboidratos simples e baixo consumo de alimentos protetores foram identificados neste estudo. Assim, essas informaçóes ressaltam a importância da educação nutricional atrelada a uma vigilância alimentar, com o intuito de evitar o aumento dos riscos relacionados à presença e desenvolvimento de doenças crônicas. 


\section{Referências Bibliográficas}

ARAÚJO, A. P. S.; BERTOLINI S. M. M. G.; JUNIOR, J. M. Alterações morfofisiológicas decorrentes do processo de envelhecimento do sistema musculoesquelético e suas consequências para o organismo. Perspectivas online - Ciências biológicas e da saúde, v. 12, n. 4, p. 22-34, jan./mar. 2014. Disponível em: <http://www.seer. perspectivasonline.com.br/index.php/biologicas_e_saude/article/viewFile/42/409>. Acesso em: 10 jan. 2017.

ARAÚJO, C. L.; MANUCUSSI e FARO, A. C. Prática de atividade física entre idosos do Vale do Paraíba, São Paulo, Brasil. Revista eletrônica trimestral de enfermagem, Murcia, n. 28, p. 223-232, out. 2012.Disponível em: <http://scielo.isciii.es/pdf/eg/v11n28/pt_ administracion3.pdf>. Acesso em: 20 jan. 2017.

AYRES, M.; AYRES Jr, M.; AYRES, D. L.; SANTOS, A. A. S. Bioestat 5.0 aplicações estatísticas nas áreas das ciências biológicas e médicas. Belém: IDSM, 2007.

BIELEMANN, R.M. Impacto da inatividade física e custos de hospitalização por doenças crônicas. Rev. Saúde Pública, São Paulo, v.49, n. 75, 2015.Disponível em: <http://www. scielosp.org/pdf/rsp/v49/pt_0034-8910-rsp-S0034-89102015049005650.pdf>. Acesso em: 25 jan. 2017.

BORGES, P. L. C.; BRETAS, R. P.; AZEVEDO, S. F.; BARBOSA, J. M. M. Perfil dos idosos frequentadores de grupos de convivência em Belo Horizonte, Minas Gerais, Brasil. Cadernos de Saúde Pública, Rio de Janeiro v.24, n.12, dez. 2010. Disponívelem:<http:// www.scielo.br/scielo.php?script=sci_arttext\&pid=S0102311X2008001200008\&lang= pt>. Acesso em: 07 out. 2016.

BUSNELLO, F. M. Aspectos nutricionais no processo do envelhecimento. São Paulo: Atheneu, 2007.

BRASIL. Ministério da Saúde. Coordenação de Saúde da Pessoa Idosa. Portal da Saúde, 25 de Abril de 2014. Disponível em: <http://portalsaude.saude.gov.br/index. php/o-ministerio/principal/secretarias/808-sas-raiz/daet-raiz/saude-da-pessoa-idosa/ 11-saude-da-pessoa-idosa/12330-apresentacao-sp-idosa>. Acesso em: 09 nov. 2016.

BRASIL. Ministério da Saúde. Secretaria de Atenção à Saúde. Departamento de Atenção Básica. Alimentação saudável para a pessoa idosa: um manual para profissionais de saúde. 1. ed. Brasília: Ministério da Saúde, 2009.

BRASIL. Ministério da Saúde. Secretaria de Atenção à Saúde. Departamento de Atenção Especializada. Manual de rotinas para atenção ao AVC. Brasília: Ministério da Saúde, 2013.

BRASIL. Ministério da Saúde. Secretaria de Atenção à Saúde. Departamento de Atenção Básica. Guia alimentar para a população brasileira. 2. ed. Brasília: Ministério da Saúde, 2014.

BRASIL. Ministério da Saúde. SISVAN - Vigilância alimentar e nutricional. Orientações básicas para coleta, processamento e análise de dados e informação em serviços de 
saúde. Brasília: Ministério da Saúde; 2004.

CAMARGOS, M. C. S.; NASCIMENTO, G. W. C.; NASCIMENTO, D. I. C.; MACHADO, C. J. Aspectos relacionados à alimentação em Instituições de Longa Permanência para idosos em Minas Gerais. Cadernos de Saúde Coletiva, Rio de Janeiro, v. 23, n. 1, p. 38-43, 2015. Disponível em: <http://www.scielo.br/pdf/cadsc/v23n1/1414-462Xcadsc-23-01-00038.pdf>. Acesso em: 09 nov. 2016.

CARVALHO, M. A.; COUTINHO, A. P. O.; CARVALHO, G. D. A.; QUEIRÓZ, D. T. G.;SANTOS, S. R. Epidemiologia dos acidentes vasculares encefálicos atendidos por meio do serviço de atendimento móvel de urgência. Revista de Enfermagem UFPE on line, Recife, v. 9, n. 3, p. 1015-1021, mar. 2015.Disponível em: <https://periodicos.ufpe.br/revistas/ revistaenfermagem/article/download/10429/11224>. Acesso em: 09 nov. 2016.

CARNEIRO, L. A. F.; CAMPINO, A. C. C.; LEITE, F. RODRIGUES, C. G.; SANTOS, G. M. M.; SILVA, A. R. A. Envelhecimento populacional e os desafios para o sistema de saúde brasileiro. Instituto de Estudos de Saúde Suplementar. São Paulo: IESS, 2013.

CORTEZ, A. C. L.; MARTINS, M. F. G. Indicadores antropométricos do estado nutricional em idosos: uma revisão sistemática. UNOPAR Científica, Ciências Biológicas e da Saúde. v. 14, n. 4 p. 271-277, 2012.Disponível em: <http://www.pgsskroton.com.br/ seer/index.php/JHealthSci/article/view/887/851>. Acesso em: 20 nov. 2016.

DOBNER, T.; BLASI T. C.; KIRSTEN, V. R. Perfil nutricional de idosos residentes em instituição geriátrica no interior do RS. Revista Brasileira de Ciências do Envelhecimento Humano. v. 1, n. 9, p. 109-118, jan./abr.2012. Disponível em: <http:// seer.upf.br/index.php/rbceh/article/view/1730/pdf>. Acesso em: 20 dez. 2016.

FECHINE, B. R. A.; TROMPIERI, N. O. O processo de envelhecimento: as principais alterações que acontecem com o idoso com o passar dos anos. InterSciencePlace, Rio de Janeiro, n. 20, v. 1, p. 106-194, 2012. Disponível em: <http://www.fonovim.com.br/ arquivos/534ca4b0b3855f1a4003d09b77ee4138-Modifica----es-fisiol--gicas-normaisno-sistema-nervoso-do-idoso.pdf>. Acesso em: 20 jan. 2017.

FISBERG, R. M.; MARCHIONI, D. M. L.; DE CASTRO, M. A.; JUNIOR, E. V.; ARÚJO, M. C.; BEZERRA, I. N.; PEREIRA, R. A.; SICHIERI, R. Ingestão inadequada de nutrientes na população de idosos do Brasil: Inquérito Nacional de Alimentação 2008-2009. Rev. Saúde Pública. São, Paulo, v. 47, sup. 1, p. 222s-230s, fev. 2013. Disponível em: <http:// www.scielo.br/pdf/rsp/v47s1/08.pdf>. Acesso em: 23nov. 2016.

FISBERG, R. M.; COLUCCI, A.C.A.; MORINETO, J.M.; MARCHIONI, D.M.L. Questionário de frequência alimentar para adultos com base em estudo populacional. Rev. Saúde Pública,São, Paulo, v. 42, n. 3, p. 550-554, abr. 2008. Disponível em: <http://www.scielo. br/pdf/rsp/v42n3/6646.pdf>. Acesso em: 15 dez. 2016.

FRANCISCO, P. M. S. B.; BELON, A. P.; BARROS, M. B. A.; CARANDINA, L.; ALVES, M. C. G. P.; GOLDBAUM, M.; CESAR, C. L. G. Diabetes auto referido em idosos: prevalência, fatores associados e práticas de controle.Cadernos de Saúde Pública, Rio de Janeiro, v. 26, n. 1; p. 175-184, jan. 2010.Disponível em: <http://www.scielo.br/pdf/csp/v26n1/18.pdf>. Acesso em: 12 dez. 2016. 
FREIRE, G. V.; Silva, I. P.; Moura, W B.; Rocha, F. C. V, Madeira, M. Z.; Amorim, F. C. M. Perfil de idosos que frequentam um centro de convivência da terceira idade.Revista Interdisciplinar. Teresina, v. 8, n. 2, p. 11-19, abr./jun. 2015. Disponível em: <http:// revistainterdisciplinar.uninovafapi.edu.br/index.php/revinter/article/view/619/ pdf_206>. Acesso em: 10 dez. 2016.

FREITAS, C. F. C.; PERALTA, A. M.; CARVALHO, A. M.; CARIOCA, A. A. F.; FISBERG, R. M.; MARCHIONI, D. M. L. Qualidade da dieta entre consumidores e não consumidores de carnes vermelhas e processadas: estudo ISA-Capital. Revista de Nutrição, Campinas, n. 28, v. 6, p. 681-689, 2015. Disponível em: <http://www.scielo.br/pdf/rn/v28n6/16789865-rn-28-06-00681.pdf>. Acesso em: 20 jan. 2016.

GOULART, D.; ENGROFF, P.; ELY, L. S.; SGNAOLIN, V.; SANTOS, E. F.; TERRA, N. L.; CARLI, G.A. Tabagismo em idosos. Revista Brasileira de Geriatria e Gerontologia, Rio de Janeiro, v. 13, n. 2, p. 313-320, 2010.Disponível em: <http://www.scielo.br/pdf/rbgg/ v13n2/a15v13n2.pdf>. Acesso em: 20 dez. 2016.

HOTT, A. M.; PIRES, V. A. T. N. Perfil dos idosos inseridos em um centro de convivência. Revista Enfermagem Integrada, Ipatinga, v. 4, n. 1, p. 765-778, jul./ago. 2011. Disponível em: <https://www.unilestemg.br/enfermagemintegrada/artigo/v4/12perfil-dos-idosos-inseridos-em-um-centro-de-convivencia.pdf>. Acesso em: 20 dez. 2016.

INSTITUTO BRASILEIRO DE GEOGRAFIA E ESTATÍSTICA - IBGE. Pesquisa de orçamentos familiares 2008-2009: análise do consumo alimentar pessoal no Brasil. Rio de Janeiro: IBGE, 2011.

- Ministério do Planejamento, Orçamento e Gestão. Sinopse do Censo Demográfico de 2010. Rio de Janeiro: IBGE, 2010A.

. Tábuas completas de mortalidade. 2010b. Disponívelem:<http://www.ibge. gov.br/home/estatistica/populacao/projecao_da_populacao/piramide/piramide. shtm>. Acesso em: 06 out. 2016.

LOHMAN, T. G. Advances in body composition assessment current issues in exercisc science series.Monograph n. 3. Champaign, IL: Human Kinetics. 1988.

MALTA, M. B.; PAPINI, S. J.; CORRENTE, J. E. Avaliação da alimentação de idosos de município paulista - aplicação do Índice de Alimentação Saudável. Ciências e Saúde Coletiva, Rio de Janeiro,v. 18, n. 2, p. 377-384, jan./fev. 2013. Disponível em: <http:// www.scielo.br/pdf/csc/v18n2/09.pdf>. Acesso em: 20 dez. 2016.

MENDES, T. A. B.; GOLDBAUM, M.; SEGRI, N.J.; BARROS, M.B.A.; CESAR, C.L.G. Diabetes Mellitus: fatores associados à prevalência em idosos, medidas e práticas de controle e uso dos Serviços de Saúde em São Paulo, Brasil. Cad. Saúde Pública. Rio de janeiro, v. 27, n. 6, p. 1233-1243, jun. 2011. Disponível em: <http://www.scielo.br/pdf/csp/ v27n6/20.pdf>. Acesso em: 20 nov. 2016. 
MILECH, Adolfo et al. Diretrizes da Sociedade Brasileira de Diabetes. São Paulo: A.C. Farmacêutica, 2016. Disponível em: <http://www.diabetes.org.br/profissionais/ images/docs/DIRETRIZES-SBD-2015-2016.pdf>. Acesso em: 20 dez. 2016.

MORAES E. N. Atenção à saúde do idoso: Aspectos conceituais. Brasília: Organização Pan-Americana da Saúde - OPAS, 2012.

NASCIMENTO, C. M.; RIBEIRO, A. Q.; SANT'ANA, L. F. R.; OLIVEIRA, R. M. S.; FRANCESCHINI S. C. C.; PRIORE S, E. Estado nutricional e condições de saúde da população idosa brasileira: revisão de literatura. Revista Médica de Minas Gerais. v. 21, n. 2, p. 174180, 2011. Disponível em: <http://rmmg.org/artigo/detalhes/195>. Acesso em: 20 jan. 2017.

ORLANDO, R.; PINHEIRO, T. L. F.; VOLKWEISH, D. S. H.; COLUSSI, E. L. Avaliação da alimentação e sua relação com as doenças crônicas não transmissíveis de um grupo de idosos de um município da região norte do estado do RS. Revista de Enfermagem Frederico Westphalen. v. 6, n. 7, p. 203-217, 2011. Disponível em: <http://revistas. fw.uri.br/index.php/revistadeenfermagem/article/view/736/1312>. Acesso em: 20 dez. 2016.

PANZIERA, F. B.; DORNELES, M. M.; DURGANTE, P. C.; SILVA, V. L Avaliação da ingestão de minerais antioxidantes em idoso. Revista Brasileira de Geriatria e Gerontologia, Rio de Janeiro. n. 14, v, 1, p. 49-58, jan./mar. 2011. Disponível em: <http://www.scielo.br/ $\mathrm{pdf} / \mathrm{rbgg} / \mathrm{v} 14 \mathrm{n} 1 / \mathrm{a} 06 \mathrm{v} 14 \mathrm{n} 1 . \mathrm{pdf}>$. Acesso em: 20 dez. 2016.

PAZ, R. C.; FAZZIO, D. M. G.; SANTOS, A. L. B. Avaliação nutricional de idosos institucionalizados. Revisa, n. 1, v. 1, p. 9-18. jan./jun, 2012. Disponível em: <http:// revistafacesa.senaaires.com.br/index.php/revisa/article/view/6/3>. Acesso em: 20 out. 2016.

PEREIRA, I. F. S.; SPYRIDES, M. H. C.; ANDRADE, L. M. B. Estado nutricional de idosos no Brasil: uma abordagem multinível. Cadernos de Saúde Pública, Rio de Janeiro, n. 32, v. 5, p. 1-12, mai. 2016. Disponível em: <http://www.scielo.br/pdf/csp/v32n5/16784464-csp-32-05-e00178814.pdf>. Acesso em: 13nov. 2016.

RIBAS, M. Atividade física para terceira idade: Discutindo a realidade dos programas no município de Ariquemes. Trabalho de conclusão de curso (Licenciatura em Educação Física)Programa Pró-Licenciatura da Universidade de Brasília - Polo Ariquemes - RO, 2013.

RIBEIRO, R. L.; TONINI, K. A. D; PRESTA, F. M. P; SOUZA, M. V. M.; PICANÇO, E. A. Avaliação nutricional de idosos residentes e não residentes em instituições geriátricas no município de Duque de Caxias/RJ. Revista Eletrônica Novo Enfoque. v. 12, n. 12, p. 39-46, 2011.Disponível em: <http://www.castelobranco.br/sistema/novoenfoque/ files/12/artigos/06.pdf>. Acesso em: 20 dez. 2016.

SANTOS, R. R.; BICALHO M. A. C.; MOTA, P.; OLIVEIRA, D. R.; MORAES, E. N. Obesidade em idosos. Revista Médica de Minas Gerais. n. 23, v. 1, p. 64-73, 2013. Disponível em: $<$ http://rmmg.org/artigo/detalhes/12>. Acesso em: 20 dez. 2016. 
SOUZA, S.B.O.; BRANCA, S.B.P. Panorama epidemiológico do processo de envelhecimento, no mundo, Brasil e Piauí: evidencias na literatura de 1987 a 2009. Enfermagem em foco. v. 2, n.3, p. 188-190, 2011. Disponível em: <http://revista.portalcofen.gov.br/index.php/ enfermagem/article/view/132>. Acesso em: 20 fev. 2017.

SCHERER, R.; SCHERER, F.; CONDE, S. R.; BOSCO, S. M. D. Estado nutricional e prevalência de doenças crônicas em idosos de um município do interior do Rio Grande do Sul. Revista Brasileira de Geriatria e Gerontologia. Rio de Janeiro, v. 16, n. 4, p. 769-779, out./dez. 2013. Disponível em: <http://www.scielo.br/pdf/rbgg/v16n4/1809-9823rbgg-16-04-00769.pdf >. Acesso em: 20 dez. 2016.

SILVA, A. K. Q, GUSMÃO; S. C., CASTRO, K. R.; MOREIRA, R. A. N.; MORAIS, A. H. A. Perfil nutricional de idosos assistidos em instituição de longa permanência na cidade de Natal, RN. Geriatria \& Gerontologia. v. 4, n. 1, p. 27-35, 2010. Disponível em: <http://ggaging. com/export-pdf/295/v4n1a05.pdf>. Acesso em: 20 dez. 2016.

SILVEIRA, M. M.;KUMPEL, D. A.; ROCHA, J. P.; PASQUALOTTI, A.; COLUSSI, E. L. Caracterização da saúde e atividade física de idosos usuários de informática. Revista Contexto \& Saúde, Rio Grande do Sul. v. 11, n. 20,p. 187-194, jan./jun, 2011.Disponível em: <https:// www.revistas.unijui.edu.br/index.php/contextoesaude/article/view/1517/1278>. Acesso em: 20 dez. 2016.

SOBREIRA, F. M. M.; SARMENTO, W. E.; OLIVEIRA, A. M. B. Perfil epidemiológico e sóciodemográfico de idosos frequentadores de grupo de convivência e satisfação quanto à participação no mesmo. Revista Brasileira de Ciências da Saúde.,v. 15 n. 4, p. 429-438. 2011. Disponível em: <http://periodicos.ufpb.br/ojs/index.php/rbcs/article/ view/10417/6860>. Acesso em: 10 dez. 2016.

SOCIEDADE BRASILEIRA DE HIPERTENÇÃO - SBH. VI Diretrizes Brasileiras de Hipertensão. Revista de Hipertensão. v. 13, n. 1 p. 1-68, jan./mar. 2010. Disponível em: <http://www. sbh.org.br/pdf/diretrizes_final.pdf>. Acesso em: 20 dez. 2016.

TAVARES, E. L.; DOS SANTOS, D. M.; FERREIRA, A. A.; MENEZES, M. F. G. Avaliação nutricional de idosos: desafios da atualidade. Revista Brasileira de Geriatria e Gerontologia. v. 18, n. 3, p. 643-650, 2015. Disponível em: <http://www.scielo.br/pdf/rbgg/v18n3/18099823-rbgg-18-03-00643.pdf>. Acesso em: 14nov. 2016.

TIRAPEGUI, J.; RIBEIRO, L. S. M. Avaliação Nutricional - Teoria e Prática. São Paulo: Guanabara Koogan, 2011.

VENTURINI, C. D.; ENGROFF, P.; SGNAOLIN, V.; EL KIK, R. M.; MORRONE, F. B.; FILHO, I. G. S.; DE CARLI, G. A. Consumo de nutrientes em idosos residentes em Porto Alegre (RS), Brasil: um estudo de base populacional. Ciência \& Saúde Coletiva. v. 20, n. 12, p. 3701-3711, 2015.Disponível em: <http://www.scielo.br/pdf/csc/v20n12/1413-8123-csc-20-12-3701. pdf>. Acesso em: 20 dez. 2016. 
ZAGO, A. S.Exercício físico e o processo saúde-doença no envelhecimento.Revista Brasileira de Geriatria e Gerontologia, Rio de Janeiro; 13(1):153-158.2010. Disponível em: <http://www.scielo.br/ pdf/rbgg/v13n1/a16v13n1.pdf $>$.Acesso em: 09 out. 2016.

WOLD HEALTH ORGANIZATION.Physical status: the use and interpretation of anthropometry. Geneva: World Health Organization, 1995. Disponível em: <http://apps.who.int/iris/bitstream/10665/37003/1/ WHO_TRS_854.pdf>. Acesso em: 09 nov. 2016. 